\title{
Nonlinear Fourier Transform: Perpetual Research Topic or Future Game-Changer?
}

Da Ros, Francesco; Gaiarin, Simone; Jones, Rasmus Thomas; Jovanovic, Ognjen; Porto da Silva, Edson; Zibar, Darko

\section{Published in:}

Signal Processing in Photonic Communications 2020

Link to article, DOI:

10.1364/SPPCOM.2020.SpTu2I.1

Publication date:

2020

Document Version

Peer reviewed version

Link back to DTU Orbit

Citation (APA):

Da Ros, F., Gaiarin, S., Jones, R. T., Jovanovic, O., Porto da Silva, E., \& Zibar, D. (2020). Nonlinear Fourier Transform: Perpetual Research Topic or Future Game-Changer? In Signal Processing in Photonic Communications 2020 [SpTu2l.1] Optical Society of America (OSA). https://doi.org/10.1364/SPPCOM.2020.SpTu2l.1

\section{General rights}

Copyright and moral rights for the publications made accessible in the public portal are retained by the authors and/or other copyright owners and it is a condition of accessing publications that users recognise and abide by the legal requirements associated with these rights.

- Users may download and print one copy of any publication from the public portal for the purpose of private study or research.

- You may not further distribute the material or use it for any profit-making activity or commercial gain

- You may freely distribute the URL identifying the publication in the public portal 


\title{
Nonlinear Fourier Transform: Perpetual Research Topic or Future Game-Changer?
}

\author{
F. Da Ros ${ }^{1}$, S. Gaiarin ${ }^{1}$, R. T. Jones ${ }^{1,3}$, O. Jovanovic ${ }^{1}$, E. P. da Silva ${ }^{1,2}$, and D. Zibar ${ }^{1}$ \\ ${ }^{\prime}$ DTU Fotonik, Technical University of Denmark, DK-2800 Kongens Lyngby, Denmark, fdro@fotonik.dtu.dk \\ ${ }^{2}$ Department of Electrical Engineering, Federal University of Campina Grande (UFCG), Paraiba, Brazil \\ ${ }^{3}$ Oticon, Smørum, Denmark
}

\begin{abstract}
The topic of nonlinear Fourier transform (NFT) will be discussed focusing on the results achieved so far, on current open challenges and on an outlook on future prospects, especially focusing on machine-learning aided NFT systems. C 2020 The Author(s)
\end{abstract}

\section{Introduction}

Optical communication systems based on single-mode fibers are currently reaching a saturation in achievable transmission rate. Kerr nonlinearity is one of the fundamental challenges to overcome to try to increase the throughput of deployed transmission systems. Whereas the last decade has seen a plethora of digital [1,2] and optical [1,3-4] methods focused on compensating for the impact of nonlinear distortion, an older mathematical theory has also been re-discovered, i.e. the inverse scattering transform recently better known as nonlinear Fourier transform (NFT) [5]. The NFT theory provides analytical solutions of the integrable nonlinear Schödinger equation (NLSE) by solving the Zakharov-Shabat spectral problem [6]. Through direct and inverse NFT, signaling schemes optimized for the dispersive and nonlinear fiber channel can be constructed, yielding to the concept of nonlinear frequency division multiplexing (NFDM) systems. Whereas this approach has been extensively explored in the past in terms of soliton and eigenvalue communications [7], current optical coherent transceivers allow overcoming a number of limitations of soliton transmission by enabling access to the full optical field. More complex signaling schemes can now be constructed, e.g. with complex modulation formats [8]. This key advance has fueled a renewed interest in the topic with several impressive demonstrations of NFDM transmission [9]. Single and dual-polarization transmission has been demonstrated for systems modulating not only the discrete (solitonic) part of the NFT spectrum [10-12] but also the continuous (dispersive) part $[13,14]$. Very recent demonstrations have shown novel methods to encode information on both spectral components for single [15] and dual-polarization [16] NFDM.

Whereas significant progress has been achieved by NFDM systems within very few years, a number of open challenges still limit their applications to practical scenarios. The NFT theory is defined for a lossless NLSE, therefore fiber loss and amplification strongly hinder the system performance limiting the transmission reach, especially for large amplifier spacing [11,17]. Furthermore, the impact of noise on NFDM systems is still not fully understood and researching optimal encoding (transmitter-side) and detection (receiver-side) strategies is an active research direction[18,19]. Finally, the vanishing boundary conditions, i.e. the need to transmit localized bursts, pose strong limitations on the achievable spectral efficiency. This limitation may be addressed by considering periodic boundary conditions with interesting preliminary results shown recently [20].

In this talk, we will review the fundamentals of NFT and NFDM systems, summarizing the state of the art, describing NFDM-specific digital signal processing techniques [21] and discussing future directions. In 2018, we reported on the use of a neural-network-based receiver for NFDM systems, showing a significant improvement in performance and/or achievable transmission reach [22]. More recently, we have shown how full end-to-end learning can be applied to NFDM systems yielding additional improvement [23]. The choice of applying end-to-end learning to NFDM is dictated by needing to restrict the vast parameter space for auto-encoders optimization in a standard coherent system. NFT provides a theoretically sound mapping between symbols and time-domain waveforms. We believe this is a valuable stepping stone towards applying full end-to-end learning to generic coherent communication systems transmitting over the nonlinear dispersive fiber channel. We will thus conclude the talk with brief prospects towards derivative-free end-to-end learning for optical communication systems.

\section{Acknowledgments}

This work was financially supported by the European Research Council through the ERC-CoG FRECOM project (grant agreement no. 771878), the Villum Young Investigator OPTIC-AI project (grant no. 29334), and the National Council for Scientific and Technological Development (CNPq), Brazil, grant 432214/2018-6. OFS Denmark is thanked for providing the low-loss fiber (TeraWave ${ }^{\circledR}$ SCUBA) used in the experiments. 


\section{References}

[1] A.D. Ellis, et al., "Performance limits in optical communications due to fiber nonlinearity," Adv.s in Opt. and Phot. 9(3), 429-503 (2017).

[2] J.C. Cartledge, et al., "Digital Signal Processing for Fiber Nonlinearities," Opt. Expr. 25(3), 1916-1936 (2017).

[3] I. Sackey, et al., "Kerr nonlinearity mitigation in 528-GBd PDM 16-QAM signal transmission over a dispersion-uncompensated link with backward-pumped distributed Raman amplification," Opt. Expr. 22(22), 2738127391 (2014).

[4] M. P. Yankov, et al., "Experimental comparison of probabilistic shaping with online PMF optimization and mid-link OPC," CLEO 2018, paper Tu4C.2.

[5] A. Hasegawa and F. Tappert, "Transmission of stationary nonlinear optical pulses in dispersive dielectric fibers. I. Anomalous dispersion," APL 23(3), 142-144 (1973).

[6] V. E. Zakharov and A. B. Shabat, "Exact theory of two-dimensional self-focusing and one-dimensional self-modulation of waves in nonlinear media," Sov. Phys. JETP 34, p. 62 (1972).

[7] A. Hasegawa, and T. Nyu, "Eigenvalue communication," JLT 11(3), 395-399, Mar 1993.

[8] M. Yousefi, and F.R. Kschischang, "Information transmission using the nonlinear Fourier transform, Part I-II-III," IEEE Trans. on Inf. Theory 60(7), 4312-4369 (2014).

[9] S.K. Turitsyn, et al., "Nonlinear Fourier transform for optical data processing and transmission: advances and perspectives," Optica 4(3), 307322 (2017).

[10] Z. Dong, et al. "Nonlinear frequency division multiplexed transmissions based on NFT," PTL 27(15), 1621-1623 (2015).

[11] S. Gaiarin, et al. "Dual-polarization nonlinear Fourier transform-based optical communication system," Optica, 5(3), pp. 263-270 (2018).

[12] W. A. Gemechu et al., "Dual polarization nonlinear frequency division multiplexing transmission," PTL 30(18) pp. 1589-1592 (2018).

[13] S.T. Le, et al., "Demonstration of nonlinear inverse synthesis transmission over transoceanic distances," JLT 34(10), 2459-2466 (2016).

[14] T. Gui, et al. "Polarization-division-multiplexed nonlinear frequency division multiplexing," in Proc. CLEO 2018, paper STu4C.3.

[15] S. T. Le, et al., "Nonlinear signal multiplexing for communication beyond the Kerr nonlinearity limit," Nat. Photon. 11, pp. 570-576 (2017).

[16] F. Da Ros et al., "Dual-polarization NFDM transmission with continuous and discrete spectral modulation," JLT 37(10), pp. 2335-2345, (2019).

[17] M Kamalian-Kopae, et al., "On the Design of NFT-Based Communication Systems With Lumped Amplification," JLT 35(24), pp. 54645472, (2017).

[18] S. Civelli, et al., "Decision-feedback detection strategy for nonlinear frequency-division multiplexing," Opt. Expr. 26(9), 12057-12071

(2018).

[19] S. Civelli et al., "Nonlinear Frequency Division Multiplexing: Immune to Nonlinearity but Oversensitive to Noise?," OFC 2020, paper W4A.7.

[20] M. Kalmalian-Kopae, et al., "Full-spectrum periodic nonlinear Fourier transform optical communication through solving the RiemannHilbert problem," JLT, pre-print.

[21] S. Gaiarin, et al., "Dual-Polarization NFDM Transmission Using Distributed Raman Amplification and NFT-Domain Equalization," PTL, 30(22), 1983-1986 (2018).

[21] R.T. Jones, et al., "Time-domain neural network receiver for nonlinear frequency division multiplexed systems," PTL 30(12), 1079-1082 (2018).

[22] S. Gaiarin, et al., "End-to-end optimized nonlinear Fourier transform-based coherent communications" CLEO 2020, paper SF2L.4. 